Article

\title{
Multiple-Code Technique for Multi-Rate Transmissions in Optical Packet Switching Networks Based on OCDMA Labels
}

\author{
Kai-Sheng Chen
}

School of Electrical and Computer Engineering, Nanfang College of Sun Yat-Sen University, Guangzhou 510970, China; 17304@mail.nfu.edu.cn or weiweibjqskwx@hotmail.com; Tel.: +86-188-140-94056

Received: 25 April 2019; Accepted: 28 May 2019; Published: 30 May 2019

\begin{abstract}
Supporting multi-rate transmission is an essential factor in current optical packet switching (OPS) networks. In this paper, the author studied a multi-rate scheme capable of forwarding packets with different signal rates based on label switching. The multiple-code (MC) technique was employed to label a packet by conveying its payload bits to multiple optical code-division multiple-access (OCDMA) labels. Spectral-amplitude-coding (SAC), which represents the chips in an OCDMA code as a set of wavelengths, was introduced to remove the multiple-access interference (MAI) from the overlapping among labels. The author tested the system effectiveness by conducting numerical analysis to formulate bit-error probability (BEP) and spectral efficiency (SE). The simulation results showed that the proposed network had a stable BEP performance when switching the packet flows of multiple data-rates.
\end{abstract}

Keywords: optical packet switching (OPS); optical-code division multiple access (OCDMA); spectral-amplitude-coding (SAC); multi-rate transmission

\section{Introduction}

In optical backbones, optical packet switching (OPS) has been widely applied due to its reduced overhead and simplified structure of network layers [1-3]. With the growing demand for internet protocol (IP) traffic, service providers not only transmit packets through a point-to-point link but also assign to a wide range of network users. OPS, providing a variety services to edge users as well as allowing users to randomly access to the other members, has been seen as a practical solution to metro-area networks (MANs) and local-area networks (LANs). Moreover, to transport data as correctly as possible, error correcting scheme of channel coding is potential for being implemented in these networks. Such coding techniques, including inserting parity bits [4] and employing iterative decoding to recover bit-interleaved signals $[5,6]$, promote the network quality to meet the demanded peak-to-average power ratios and acceptable information loss under given physical constraints. However, optical buffering acts a limitation in OPS, which comes from the immaturity of converting the electrical memory scheme of random access memory (RAM) into the optical domain. This drawback can be partially circumvented by employing optical burst switching (OBS) [7] supporting bufferless networks or using fiber delay lines (FDLs) as memory units [8].

The packet switching proposal in OPS, where labels are processed in the optical domain, enables service providers to manage the packet flows more efficiently. As most of the key functions are optically executed, the bottleneck of requiring optical-to-electrical and electrical-to-optical conversions can be alleviated. The conversions performed by the electronic components in routers limit the switching speed and consume considerable power. Therefore, employing OPS to achieve high-quality networks has become a trend in optical communications. OPS maps packet labels into physical layer features 
such as the time or wavelength of optical signals or optical codes. Core routers recognize the labels to enable a packet to travel along an established link between the edge routers. The link normally includes multiple label switched paths (LSPs). The LSP information represented as optical labels is assigned to a packet before it enters the network.

To identify the overlapping labels in the same optical bandwidth, a multiplexing technique is required at core routers. Optical code-division multiple access (OCDMA), providing high speed and security communications in access networks, has the potential to support all-optical processing [9-11]. The obtained label numbers in OCDMA are more flexible than the ones in wavelength-division multiplexing (WDM), and recent literature has demonstrated that OCDMA is compatible with the labeling scheme in OPS [9-12]. Converting packet labels into optical codes can be categorized into two methods, the explicit and implicit labels, according to the packet structure [12]. For explicit labels, edge routers attach the encoded optical signals to a packet's head. If the core routers fail to recognize the labels correctly, it results in great degradation as the entire packet drops. For implicit labels, each payload bit in a packet is programmed into an OCDMA signal. It reaches a high transmission efficiency as the implicit labels and the payload are located in the same time slots or bandwidth. The incorrectly decoded labels in a specific payload bit can be compensated by the correctly decoded labels carried by other bits in the same packet, and the lost payload bit is reduced. Therefore, the author preferred to employ implicit labels to better fit the labeling mechanisms in OPS.

With the increased stability of fiber channels and the emergence of multimedia services over the Internet, network users expect service providers to support communications with different classes of quality-of-service (QoS). In typical optical communication systems, multi-service transmissions mainly focus on differentiated bit-error probabilities (BEPs) $[13,14]$. BEP values can be varied by performing digital signal processing (DSP) at the decoder [13] or modifying the optical signals [14]. In current OPS, except for BEP, different demands of signaling rates are required to exist together in the same network.

In this paper, the author introduced a multi-rate switching strategy in generalized multi-protocol label switching (GMPLS) by employing the multiple-code (MC) technique [15]. In the proposed scheme, the signal rate of packet flow is varied with the number of OCDMA labels carried by a packet. Spectral-amplitude-coding (SAC), which represents the chips in an OCDMA code as a set of wavelengths, was introduced to remove the multiple-access interference (MAI) from the overlapping among labels. The proposed architecture was capable of providing arbitrary classes of bit-rates rather than a simple two-class scenario. The label assignments to core routers and the packet structure were demonstrated in detail. Furthermore, except for the bit-error probability (BEP), the author derived the network spectral efficiency (SE) to describe the network characteristics more adequately.

The rest of this paper is structured as follows. In Section 2, typical multi-rate schemes in OCDMA systems are reviewed. In Section 3, the author covers concepts of multi-rate switching in OPS and demonstrates the packet structure in time and wavelength domain. Hardware implementations of generating and detecting OCDMA labels in multi-rate transmissions are discussed in Section 4. Section 5 focuses on the performance analysis of network BEP and SE. The last section brings a conclusion.

\section{Related Works}

Several schemes of providing multi-rate transmissions in OCDMA have been proposed [15-17]. In [16], users sent data bits as multi-length variable weight (MLVW) sequences based on their requested services. Multi-rate service is achieved by selecting codes with various lengths and weights, where the high-weight and low-length codes are employed for the packets with a higher bit rate. Another multi-rate OCDMA scheme is known as multi-code keying (MCK) [17]. Instead of the one-on-one mapping of a data bit to a code sequence, several bits are included in a code symbol in MCK where the data rates can be switched by assigning different bit numbers to a symbol. The concept of MC is to express a serial bit stream into $M$ parallel branches. Each parallel bit is encoded with a specific OCDMA code. Then, $M$ codes are multiplexed in a shared channel and transmitted simultaneously. Receivers recover the bit sequence by serial-to-parallel conversion after the multiplexed signals are 
decoded. Users are able to transmit data with $M$ times of the basic bit rate by setting different numbers of parallel branches and codes.

Compared with the previously mentioned multi-rate schemes MLVW and MCK, MC has several advantages. First, the encoder/decoder structure for generating/recognizing OCDMA labels can be unified, while several codec designations are required for supporting MLVW labels with various code lengths and code weights. Furthermore, MC outperforms MCK in network flexibility. The gains of bit-rate in $\mathrm{MC}$ can be scaled to any arbitrary positive integers $M$, while the gains in MCK are limited to discrete values of $2^{M}$. The code utilization in MC is also more efficient. To achieve $M$ times of the basic bit rate, up to $2^{M}$ codes are required to express a symbol of $M$ bits in MCK, while only $M$ codes are sufficient to transmit $M$ bits in the basic duration for MC.

However, when multiple codes are used for high-rate transmissions, multiple-access interference (MAI) is accumulated from the overlapping label signals. Detection errors will increase significantly if the MAI is over an acceptable threshold at the decoder. Therefore, the author adopted spectral-amplitude-coding (SAC) [18-20] to eliminate the MAI effect. Combining the characteristics of SAC codes and an interference cancellation algorithm known as balanced detection, MAI can be blocked at the receiver. In the existing research, the investigations in the multi-rate code-switched network have been limited to phase-coding and time-spreading OCDMA. Therefore, the contribution of this paper is to analyze the performance of multi-rate switching based on SAC labels.

\section{Network Topology and Label Processing Scheme for Multi-Rate Transmissions}

The proposed network topology includes edge routers connected via core routers supporting code switching, as shown in Figure 1. Before the packets are transferred, an end-to-end link consisting of multiple LSPs is established by the edge routers at two link edges. Label stacking is presented to simplify the switching scheme. At the edge router, labels corresponding to LSPs along the link are aggregated into multiplexed OCDMA signals. For the entire route, the label numbers and the label codes in the stack are fixed and do not vary with the LSPs traveled by the packet. This function enables the core routers to avoid label swapping and label inserting during the switching procedure, as the packet traffic is only guided by identifying the stacked labels. The core routers implemented along the link manages the packet switching among LSPs. The packets are switched from a previous LSP to a new one according to the decoded signals of SAC labels. In the network, each LSP is mapped into a code set to allow for the possible transmissions of different bit-rates. The code number assigned to a packet is based on the desired class of signaling rate. Although the mapping scheme restricts the label distributing region of core routers, it has a benefit in being capable of multi-rate switching.

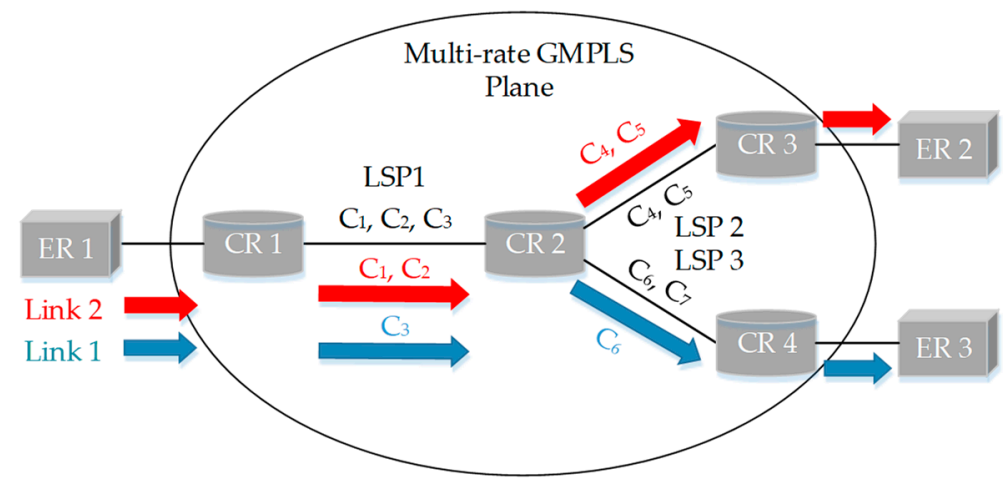

Figure 1. Proposed optical packet switching (OPS) topology and multi-rate operation. CR: core router; ER: edge router; GMPLS: generalized multi-protocol label switching; LSP: label switching path.

\subsection{Label Assignment}

In the exemplary multi-rate OPS network in Figure 1, it is assumed that the network was able to support two classes of the requested bit-rates. For the high-rate users of class 2, they were assigned 
with two codes in a LSP, while only a single code was distributed to the low-rate users of class 1. The author established two packet-switching links, where the bit-rate of link 2 was twice than that of link 1. The first path in link 2 was LSP 1 , which mapped labels $C_{1}, C_{2}$, and $C_{3}$. Therefore, two labels, $C_{1}$ and $C_{2}$, were assigned to the packets of class 2 , while the packets of class 1 carried the remaining label $C_{3}$. In the second path in link 2 (LSP 2), the packets in class 2 selected two codes, $C_{4}$ and $C_{5}$, as the attached labels. For LSP 3, although two labels were mapped, only one of them $\left(\mathrm{C}_{6}\right)$ was employed to forward the low-rate packets in this path. Based on the above switching mechanism, a stack of two and four labels was inserted to the packets of class 1 and class 2 at edge router 1, respectively. A packet of class $m$ travelling $p$ LSPs carried $m p$ labels in this scheme.

The label stacks of the two-class transmissions are expressed as vectors in Figure 2. The author used balanced incomplete block design (BIBD) codes [21] to label packets of all classes. A cyclic relation exists between any two BIBD codes of the same length, where $\mathrm{C}_{k}=\mathrm{T}^{(k-1)} \mathrm{C}_{1}$ for $1 \leq k \leq L$, and $\mathrm{T}$ is the operator that cyclically shifts a vector to a right element. In this example, BIBD codes of length $L=7$, weight $w=3$, and in-phase cross-correlation $\lambda=1$ were employed as packet labels. The high-rate users were labeled with $C_{1}, C_{2}, C_{4}$, and $C_{5}$, while the low-rate users were labeled with $C_{3}$ and $C_{6}$.

\begin{tabular}{|c|c|ccccccc|}
\hline \multicolumn{1}{|c|}{ LSP } & Label & \multicolumn{8}{|c|}{ Code-word } \\
\hline \multirow{3}{*}{ LSP 1 } & $\mathrm{C}_{1}$ & 1 & 1 & 0 & 1 & 0 & 0 & 0 \\
\cline { 2 - 9 } & $\mathrm{C}_{2}$ & 0 & 1 & 1 & 0 & 1 & 0 & 0 \\
\hline \multirow{2}{*}{ LSP 2 } & $\mathrm{C}_{3}$ & 0 & 1 & 0 & 0 & 0 & 1 & 1 \\
\hline & $\mathrm{C}_{4}$ & 1 & 0 & 0 & 0 & 1 & 1 & 0 \\
\hline \multirow{2}{*}{ LSP 3 } & $\mathrm{C}_{5}$ & 0 & 0 & 0 & 1 & 1 & 0 & 1 \\
& $\mathrm{C}_{6}$ & 0 & 0 & 1 & 1 & 0 & 1 & 0 \\
\hline
\end{tabular}

Figure 2. Label distribution of two-class transmission.

Figure 3 shows the packet structure with stacked SAC labels for high- and low-rate transmissions. The payload bits are sent as a train of optical pulses modulated with SAC codes using the on-off keying (OOK). If the payload bit " 1 " is transmitted, a signal of the label stack representing the packet's link appears. On the other hand, if bit " 0 " has been declared, there is no signal during the bit interval $T_{\mathrm{b}}$. For high-rate users, there are two bits in $T_{\mathrm{b}}$. The former and the latter bit are conveyed to the first and the second label assigned to an LSP, respectively. The labels are overlapped in time and occupy the same bit duration as the low-rate users. When the high-rate input bits are " 11 ", as the LSP number is two, each bit carries two labels, resulting in a total of four codes being simultaneously transmitted in $T_{\mathrm{b}}$.

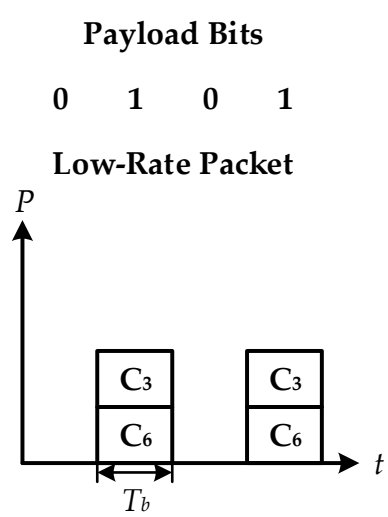

\section{Payload Bits}
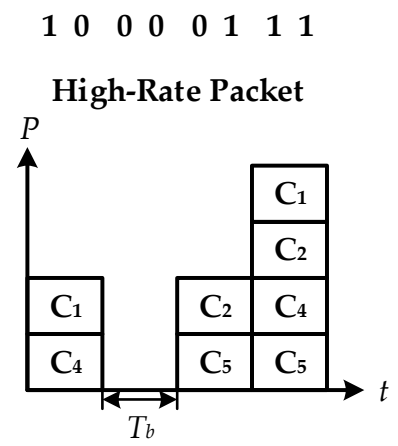

Figure 3. Optical packet structure with stacked labels for high-rate and low-rate users.

In a SAC labeling scheme, each label is expressed as a binary code $C_{k}$ with chip elements selected from $\{1,0\}$. The encoder prints the code onto a light source's spectrum by dividing it into $L$ wavelengths 
and multiplying each wavelength with $c_{k}(i)$, where $c_{k}(i)$ is the $i$-th chip element of $C_{k}$ and $1 \leq i \leq L$. Then, the encoded labels are aggregated to form label stacking. In each $T_{\mathrm{b}}$, the label number in the stack is dependent on the payload bits and the LSP number in a switching link. Figure 4a,b show the encoded spectrum when the low-rate bit is " 1 ", and the high-rate bits are " 11 ", respectively.

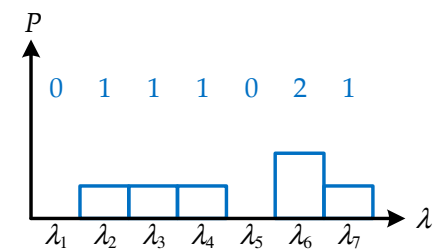

(a)

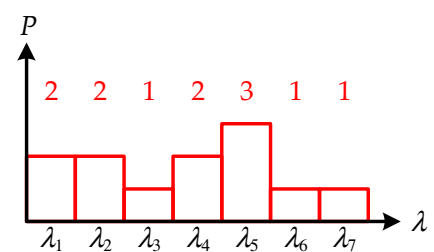

(b)

Figure 4. Spectrum of label stack for (a) low-rate; (b) high-rate packet.

\subsection{Label Identification}

In the proposed label-based network, the packet switching path is determined by executing label identification at the core routers. The correlation properties of BIBD codes are employed to decode the desired labels from the label stack without MAI. The correlation function between two BIBD codes, $\mathrm{C}_{k}$ and $\mathrm{C}_{h}$, can be found in the following expressions:

$$
R(\tau)=\sum_{i=1}^{L} c_{k}(i) c_{h}(i)=\left\{\begin{array}{cc}
w, & \tau=0 \\
1, & \tau \neq 0
\end{array}\right.
$$

where $\tau=k-h$ and $-L+1 \leq \tau \leq L+1$. Complementary code $\overline{\mathrm{C}}_{k}$ is obtained by replacing each chip element $c_{k}(i)$ in $C_{k}$ into $1-c_{k}(i)$. The correlation property of $\bar{C}_{k}$ and $C_{h}$ is:

$$
S(\tau)=\sum_{i=1}^{L} \bar{c}_{k}(i) c_{h}(i)=\left\{\begin{array}{cc}
0, & \tau=0 \\
w-1, & \tau \neq 0
\end{array}\right.
$$

where $\bar{c}_{k}(i)$ is defined as $1-c_{k}(i)$. Based on the above properties, a decoder can execute the following operation to eliminate MAI resulting from the overlapping of label signals:

$$
R(\tau)-\frac{1}{w-1} S(\tau)=\left\{\begin{array}{cc}
w, & \tau=0 \\
0, & \tau \neq 0
\end{array}\right.
$$

The function of Equation (3) is known as balanced detection. Figure 5 shows the correlation functions of BIBD codes of $w=3$ and $L=7$. There is an auto-correlation peak when two of the exact same BIBD codes are correlated with each other. When two codes are mismatched, the function values of balanced detection are always zero.

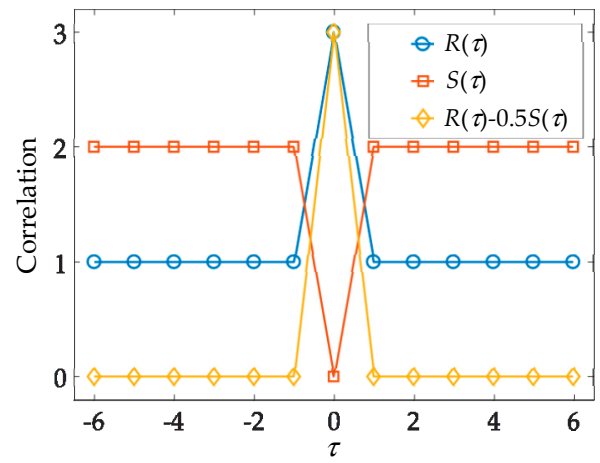

Figure 5. Plots of three correlation functions of BIBD codes of $w=3$ and $L=7$. 
Table 1 shows the procedures of label identification and MAI elimination for two classes of packets at core router 1 in Figure 1 . Here, balanced detection acts as a method to determine the switching path based on the information carried in the label stack. At the decoder, the stacked label vector $S=$ $(s(1), s(2), \ldots, s(L))$ is correlated with label code $C_{k}$ for label recognition. For low-rate packets, it is assumed that LSP 1 is mapped to $C_{1}$. If decoder $C_{1}$ generates a high-amplitude pulse proportional to the code weight $(w=3)$ at its output, it means that $C_{1}$ is included in the stack. Therefore, the core router recognizes LSP 1 as the switching path and sends the packet to the correct port, as shown in Table 1. To transmit packets of different classes, the decoders are required to detect all of the labels assigned to that LSP. For example, the path connection of LSP 1 for high-rate packets is established by decoding $\mathrm{C}_{2}$ and $\mathrm{C}_{3}$, as shown in Table 2 .

Table 1. Label identification for multi-rate switching at core router 1 for the low-rate user.

\begin{tabular}{|c|}
\hline 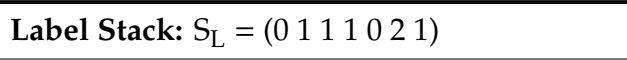 \\
\hline $\begin{array}{l}\sum_{i=1}^{7} s_{\mathrm{L}}(i) c_{3}(i)=0+1+0+0+0+2+1=4 \\
\sum_{i=1}^{7} s_{\mathrm{L}}(i) \bar{c}_{3}(i)=0+0+1+1+0+0+0=2 \\
\sum_{i=1}^{7} s_{\mathrm{L}}(i) c_{3}(i)-\frac{1}{2} \sum_{i=1}^{7} s_{\mathrm{L}}(i) \bar{c}_{3}(i)=4-1=3\end{array}$ \\
\hline Packet Switching Path: LSP 1 \\
\hline
\end{tabular}

Table 2. Label identification for multi-rate switching at core router 1 for the high-rate user.

\begin{tabular}{|c|c|}
\hline 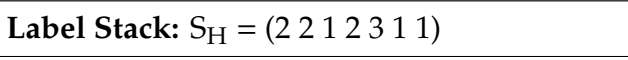 & \\
\hline $\begin{array}{l}\sum_{i=1}^{7} s_{\mathrm{H}}(i) c_{1}(i)=2+2+0+2+0+0+0=6 \\
\sum_{i=1}^{7} s_{\mathrm{H}}(i) \bar{c}_{1}(i)=0+0+1+0+3+1+1=6 \\
\sum_{i=1}^{7} s_{\mathrm{H}}(i) c_{1}(i)-\frac{1}{2} \sum_{i=1}^{7} s_{\mathrm{H}}(i) \bar{c}_{1}(i)=6-3=3\end{array}$ & $\begin{array}{l}\sum_{i=1}^{7} s_{\mathrm{H}}(i) c_{2}(i)=0+2+1+0+3+0+0=6 \\
\sum_{i=1}^{7} s_{\mathrm{H}}(i) \bar{c}_{2}(i)=2+0+0+2+0+1+1=6 \\
\sum_{i=1}^{7} s_{\mathrm{H}}(i) c_{2}(i)-\frac{1}{2} \sum_{i=1}^{7} s_{2}(i) \bar{c}_{1}(i)=6-3=3\end{array}$ \\
\hline Packet Switching Path: LSP 1 & Packet Switching Path: LSP 1 \\
\hline
\end{tabular}

\section{Hardware Design of Codec for Multi-Rate Labels}

Figure 6 shows the architecture of packet generation for the proposed multi-rate network. For payload bits of class $m$, the serial bit stream is divided into $m$ parallel streams. Here, it is assumed that the network supports up to $M$ different signal rates and $1 \leq m \leq M$. An encoder array optically labels each bit in the parallel branch with a set of optical codes. The code set consists of $p_{m}$ labels, where $p_{m}$ is the LSP number in the end-to-end path for the packet of class $m$. Here, a label is denoted as $\mathrm{C}(x, y, z)$, where $x$ is the user class, $y$ is the parallel branch number, and $z$ is the number of LSPs, respectively. For label generation, each packet is sent to a given number of encoder arrays according to its class of signal rate. The labels of the lowest rate packets are formed by a single encoder array. The high-rate packets with $m$ times of the lowest rate are formed by $m$ encoder arrays. After labeling, packets of various classes are aggregated and forwarded to the network. 


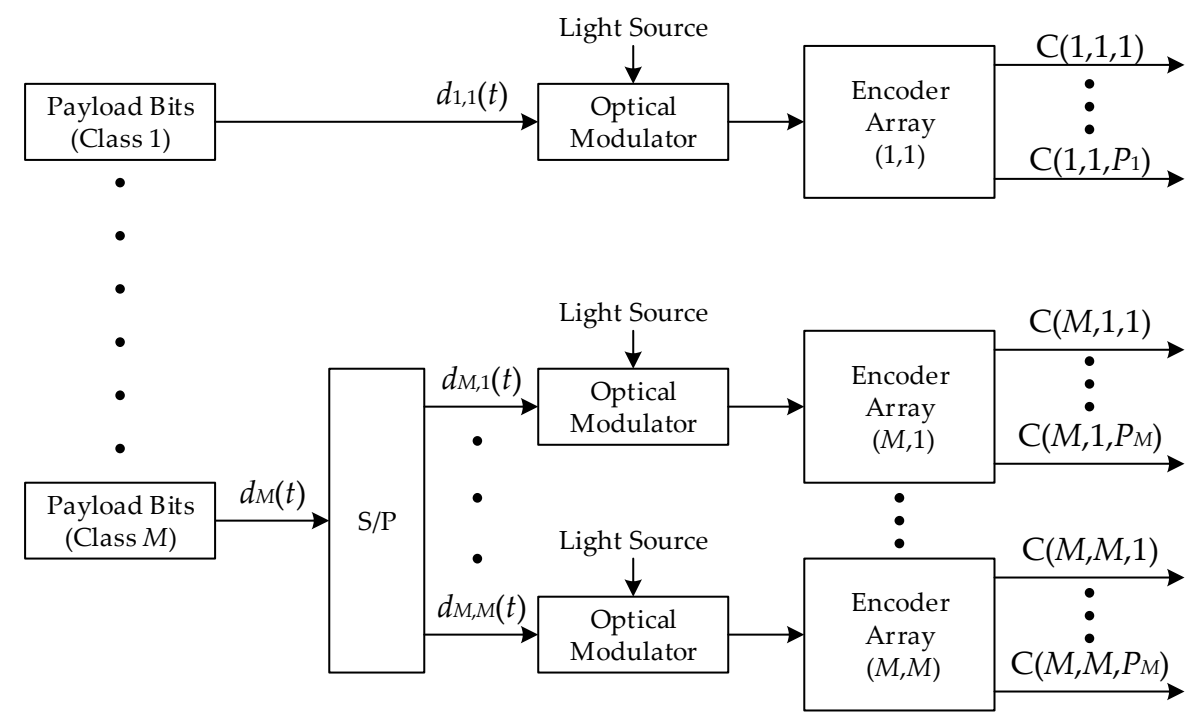

Figure 6. Optical packet generation for multi-rate network.

Figure 7 shows a BIBD encoder for creating a SAC label. An encoder array of class $m$ in Figure 5 contains $P_{m}$ encoders that generate $P_{m}$ labels for a payload bit. Each encoder is made up of $w$ fiber Bragg gratings (FBGs) and an optical combiner. A light source with a bandwidth of $L$ wavelengths is sent to FBGs of central wavelengths $\lambda i$, where $i$ is the position of the chip " $1 \mathrm{~s}$ " in a BIBD code vector.

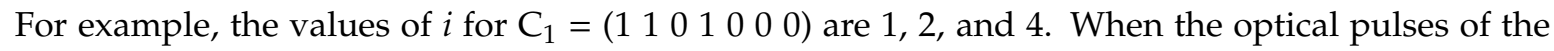
light source enter a FBG, the wavelength component matching the central wavelength is reflected. The wavelength components reflected from three FBGs, $\lambda_{1}, \lambda_{2}$, and $\lambda_{4}$, represent the SAC label encoded on the optical spectrum. The unused wavelengths corresponding to chip " $0 \mathrm{~s}$ " are not collected by the encoder as they pass through the FBGs.

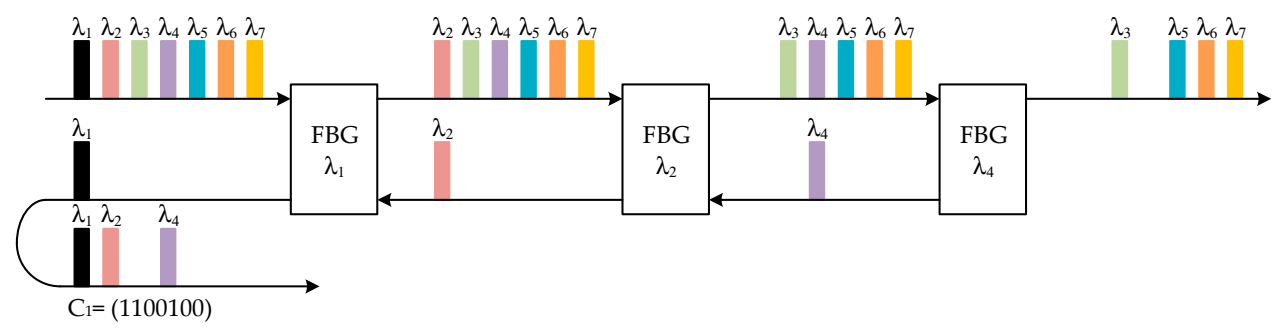

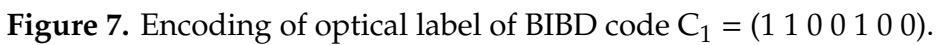

Implementing multi-rate transmission with multi-code labels requires the deployment of OCDMA decoders in the router architecture. The key function of label processing is to realize the optical codes carried by packets of various data rates. Figure 8 shows the architecture of a core router capable of packet switching over the network of multi-rate label assignment. At each core node, the aggregated packet flow is divided and sent to $M$ decoder arrays for label decoding. While the label identification is processed, the packet stays in the optical domain. When a packet is switched at a core router, the fiber delay line (FDL) in Figure 8 can be seen as a replacement of an optical buffer. The queue of packet achieving by the FDL is required to compensate the label processing time. Provided with the decoded result, an optical cross-connect (OXC) switches the packet to the destined LSP based on the label information in the stack. The decoder structure for identifying a BIBD label is similar to that of the encoder, as shown in Figure 9. The primary purpose is to calculate the correlation value of the wavelength train of the label stack with the wavelength-domain function of cascade FBGs. The implementation of FBGs is in the reversed order to compensate for the traveling time of wavelengths in the encoder. An additional attenuator of $1 /(w-1)$ is attached so that the decoder operation is the same as Equation (3). The balanced detector ensures that the MAI of the unrelated 
labels is blocked. The original payload bit is restored from the wavelength signals to the data pulse in the time-domain.

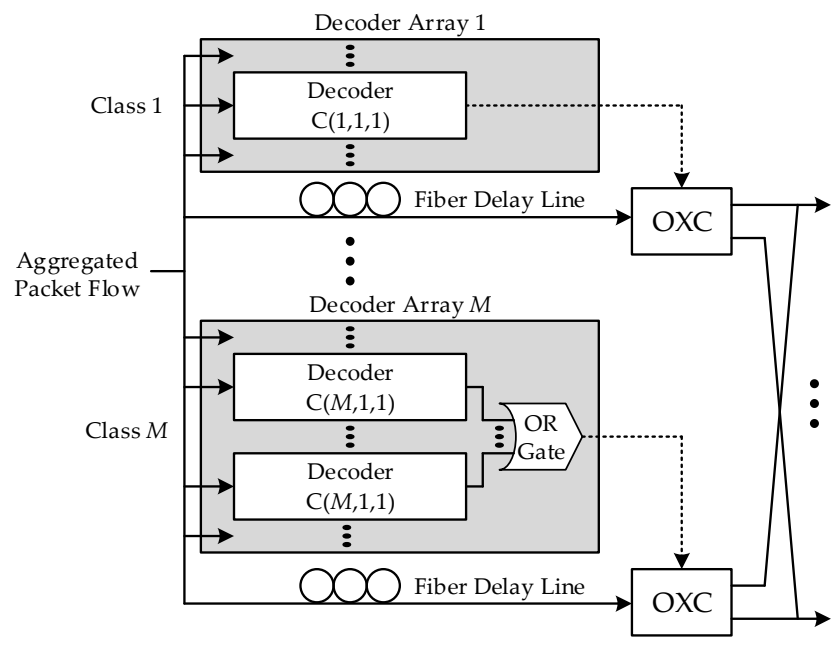

Figure 8. Optical packet processing for multi-rate network.

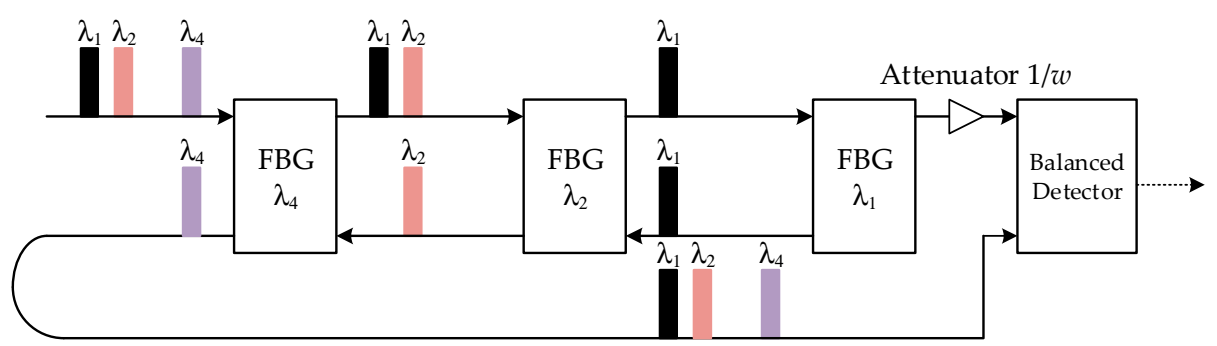

Figure 9. Decoding of optical label code.

\section{Performance Analysis and Discussion}

In this section, the author considers the variations of the decoded signal at the core routers due to the phase-induced intensity noise (PIIN) and thermal noise in the optical-electrical conversion of the balanced detector. As all packets of different classes were generated by a standard light source, it was assumed that each label reaching the decoder at core routers had equivalent power. In the following analysis, the author employed an unpolarized light source with the flat power spectral density (PSD) of $P_{\mathrm{sr}} / \Delta v$ over $\Delta v$. Here, $P_{\mathrm{sr}}$ denotes the effective power received by the decoder in $\mathrm{dBm}$ and $\Delta v$ is the light source bandwidth in Hz. At the output of a specific decoder, the average photo-current of a decoded BIBD label when the payload bit is " 1 " is expressed as:

$$
I_{1}=R P_{\mathrm{sr}} w / L
$$

where $R$ is the responsivity of the photo-diodes in the balanced detector. Given the condition that $K$ labels simultaneously enter the decoder, the average PIIN power is [21]:

$$
\left\langle I_{\mathrm{PIIN}}^{2}\right\rangle=\frac{B R^{2} P_{\text {sr }}^{2}}{\Delta v L}\left\{w+\left[3+\frac{1}{w-1}\right](K-1)+\frac{w}{L}\left[1+\frac{1}{w-1}\right](K-1)(K-2)\right\}
$$

where $B$ is the electrical bandwidth. The total label number $K$ is defined as $\sum_{m=1}^{M} m p_{m}$ when the user sends payload bits " 1 " and " 0 " with equal probability in a packet. The BEP for the payload bit encoded with a single label can be obtained by employing the following equation: 


$$
B E P=\frac{1}{2} \operatorname{erf} c\left[\frac{I_{1}}{\sqrt{8\left(\left\langle I_{\mathrm{PIIN}}^{2}\right\rangle+\left\langle I_{\mathrm{th}}^{2}\right\rangle\right)}}\right]
$$

where $\left\langle I_{\text {th }}^{2}\right\rangle$ is the variance of the thermal noise. For the packets of class $m$, as $m$ labels are multiplexed in a basic time duration $T_{\mathrm{b}}$, the BEP for the packets is expressed as $\mathrm{BEP}_{m}=1-\left(1-\mathrm{BEP}^{m}\right)$.

Figure 10 shows the relationship between BEP and the class of user bit-rates. Parameters used for the simulations were $R=0.85 \mathrm{~A} / \mathrm{W}, P_{\mathrm{sr}}=-10 \mathrm{dBm}, B=100 \mathrm{MHz}$, and $\Delta v=3.75 \mathrm{THz}$, respectively. For packets of class $m$, the bit-rate was set at $m R_{\mathrm{b}}$, where $R_{\mathrm{b}}$ was the lowest bit-rate of class 1 and $R_{\mathrm{b}}=155 \mathrm{Mb} / \mathrm{s}$. Here, five classes of packets using BIBD codes of $w=9$ and $L=73$ as labels were simultaneously transmitted and decoded at the core routers. It was assumed that the switching paths for all classes of packets had the same LSP number, $p_{1}=p_{2}=\ldots=p$. For a given LSP number $p$, the BEPs of the five classes are nearly constant, and the variations of performance among classes can be neglected. The result revealed that the proposed scheme provides an OPS network with a stable function of switching multi-rate packets. Moreover, LSP numbers of 2,3, and 4 were demonstrated in this figure for comparison. The case of $p=2$ reached the smallest BEPs, as a packet carries fewer labels to travel the link with a small LSP number.

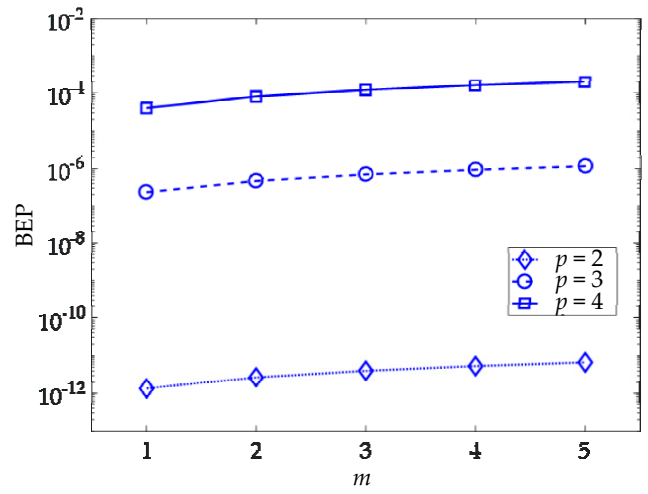

Figure 10. Bit-error probability (BEP) versus the user class of bit-rates $m$ for different LSP numbers.

The relationship between BEP and the LSP number $\mathrm{p}$ for different user distributions is shown in Figure 11. The author analyzed three cases of a two-user scenario, where the differences among the cases were the user numbers in class 1 and 2 . Given a fixed user number $(K=2)$, the BEPs of class 2 $\left(K_{2}=2\right)$ were higher than those of class $1\left(K_{1}=2\right)$, where $K_{m}$ is the user number in class $m$, and $m=1,2$. The BEPs for the hybrid scheme, where users of both classes coexist $\left(K_{1}=K_{2}=1\right)$, are in the middle of the pure high-rate and the pure low-rate schemes. As high-rate users require more labels to perform packet switching under the same switching path, they suffer more considerable noise power. Furthermore, since PIIN variance is positively related to the LSP number, the performance becomes worse as $p$ increases.

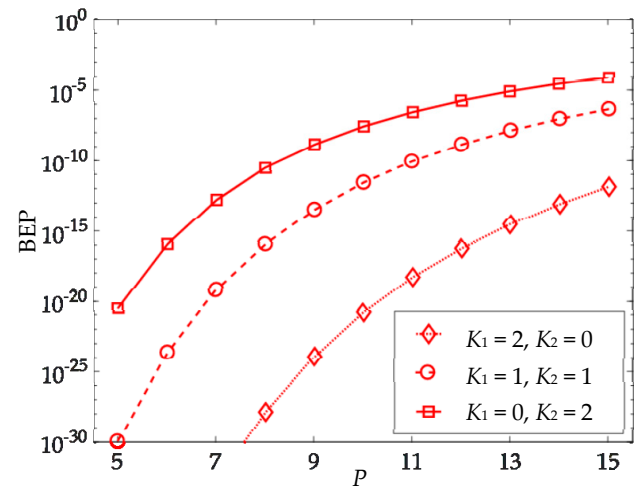

Figure 11. BEP versus the LSP number $p$ for different user distributions. 
Shown in Figure 12 are the curves of the user class $m$ versus LSP number $p$ of three cases of BEPs. Based on the previously mentioned analysis, BEP is determined by the total label number $K$. When a single-class scenario is considered, $K$ is the product of $m$ and $p$, so one parameter is inversely proportional to the other. The figure shows that a packet travels a longer LSP at the expense of the reduced signal rate.

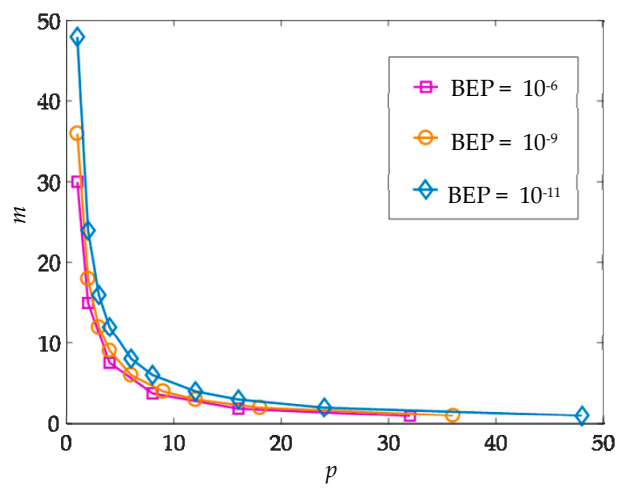

Figure 12. User class $m$ versus the LSP number $p$ for different BEPs.

Figure 13 shows the relationship between spectral efficiency (SE) and the LSP number $p$, and three cases of BEPs were compared. SE, a performance index to evaluate bit-rates for a given BEP, has the following definition [22]:

$$
S E=\frac{\text { Aggregate Data Rate }}{\text { Light Source Bandwidth }}=\frac{K R_{b}}{\Delta v}
$$

It can be seen in Figure 13 that SE increased with the required BEP. This is because more users are allowed to transmit data when the network increases the acceptable level of noise power. When the LSP number $p$ is small, a better SE is yielded at the expense of the reduced switching path. The tradeoff between SE and the distance of packet switching requires careful considerations when the network is designed. Note that SE is independent of class number $m$. When a different value $m$ is assigned, the available user number and the bit-rate become $K / \mathrm{m}$ and $m R_{\mathrm{b}}$, respectively. However, the result of SE in Equation (3) remains unchanged as the product of these two parameters is a constant.

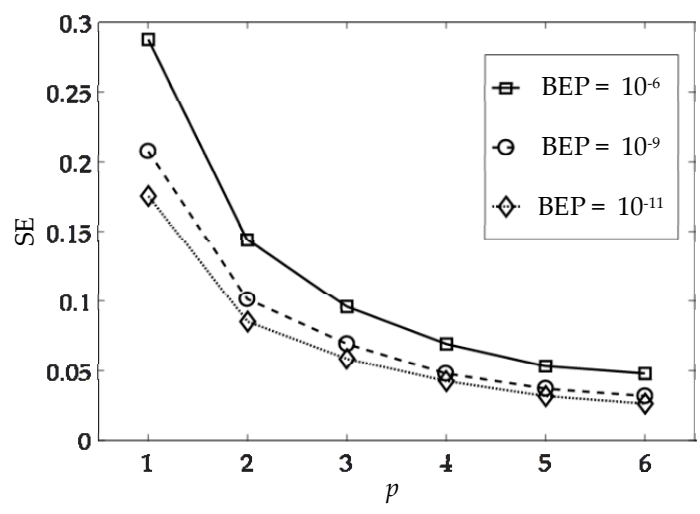

Figure 13. SE versus the LSP number $p$ for different BEPs.

\section{Conclusions}

In this paper, the author investigated a multi-rate OCDMA scheme to meet the requirements of multimedia services in current OPS networks. A packet switching system based on the multi-code technique was proposed where the packets of various bit-rates were combined and transmitted simultaneously. Numerical examples of packet label encoding and decoding were demonstrated to validate the multi-rate models. In addition, the author described the packet structure and the hardware 
implementation of the routers. Some performance indexes such as the BEP and SE were derived from the mathematical formula modeling the photo-current and the noises of the decoded labels. Based on the simulation results, the network BEP is a constant function of the user class $m$, which implies that packets of various data rates can be switched with the benefit of stable performance. Moreover, an analysis of the combinations of user numbers among bit-rate classes was conducted. The findings showed that high-rate users were more sensitive to the changes in LSP numbers, as more labels were required to switch the packets along a given link compared with the low-rate ones. A large number of overlapping labels induced a higher noise power at the decoder and BEP was increased. Finally, the SE of the proposed network was analyzed and the tradeoff between SE and the switching path distance was demonstrated.

Designing a hybrid model that integrates two or more multi-rate techniques is an exciting topic. By considering the pros and cons of each scheme, the hybrid network is flexible to meet the various requirements of operating environments. For future works, the author aims to extend the independent MC design in OPS to a collective one. The user assignment to each multi-rate scheme, the structure of optical encoders/decoders, and the multi-rate switching strategies in OPS require further investigations.

Funding: This research received no external funding.

Conflicts of Interest: The author declares no conflict of interest.

\section{References}

1. Segawa, T.; Ibrahim, S.; Nakahara, T.; Muranaka, Y.; Takahashi, R. Low-power optical packet switching for 100-Gb/s burst optical packets with a label processor and $8 \times 8$ optical switch. J. Lightwave Technol. 2016, 34, 1844-1850. [CrossRef]

2. Zhao, Z.; Wu, B.; Li, B.; Xiao, J.; Fu, S.; Liu, D. Multihop routing enabled packet switching with QoS guarantee in optical clos for data centers. J. Opt. Commun. Netw. 2018, 10, 624-632. [CrossRef]

3. Argibay-Losada, P.J.; Yoshida, Y.; Maruta, A.; Schlosser, M.; Kitayama, K.I. Performance of fixed-length, variable-capacity packets in optical packet-switching networks. J. Opt. Commun. Netw. 2015, 7, 609-617. [CrossRef]

4. McEliece, R. Practical codes for photon communication. IEEE Trans. Inf. Theory 1981, 27, 393-398.

5. Matuz, B.; Paolini, E.; Zabini, F.; Liva, G. Non-binary LDPC code design for the Poisson PPM channel. IEEE Trans. Commun. 2017, 65, 4600-4611. [CrossRef]

6. Lapidoth, A.; Moser, S.M. On the capacity of the discrete-time Poisson channel. IEEE Trans. Inf. Theory 2008, 55, 303-322. [CrossRef]

7. Hu, H.; Ji, H.; Pu, M.; Galili, M.; Yvind, K.; Oxenløwe, L.K. 160-Gb/s silicon all-optical packet switch for buffer-less optical burst switching. J. Lightwave Technol. 2015, 33, 843-848. [CrossRef]

8. Lim, H. Number of tunable wavelength converters and internal wavelengths needed for cost-effective design of asynchronous optical packet switching system with shared or output fibre delay line buffer. IET Commun. 2013, 7, 1419-1429. [CrossRef]

9. Chen, K.S. Label stacking scenarios in hybrid wavelength and code-switched GMPLS networks. Electronics 2018, 7, 251. [CrossRef]

10. Beyranvand, H.; Salehi, J.A. All-optical multiservice path switching in optical code switched GMPLS core networks. J. Lightwave Technol. 2009, 27, 2001-2012. [CrossRef]

11. Chen, K.S.; Chen, Y.C.; Liao, L.G. Advancing high-speed transmissions over OCDMA networks by employing an intelligently structured receiver for noise mitigation. Appl. Sci. 2018, 8, 2408. [CrossRef]

12. Seddighian, P.; Ayotte, S.; Rosas-Fernandez, J.B.; Penon, J.; Rusch, L.A.; LaRochelle, S. Label stacking in photonic packet-switched networks with spectral amplitude code labels. J. Lightwave Technol. 2007, 25, 463-471. [CrossRef]

13. Ahmed, M.; Ahmad, I.; Habibi, D. Service class resource management for green wireless-optical broadband access networks (WOBAN). J. Lightwave Technol. 2015, 33, 7-18. [CrossRef]

14. Yang, C.C.; Chen, K.S.; Huang, J.F.; Kuo, J.C. Differentiated service in bidirectional radio-over-fiber system over spectral-amplitude-coding OCDMA network. Photonics 2016, 3, 53. [CrossRef] 
15. Chang, C.Y.; Chen, H.T.; Yang, G.C.; Kwong, W.C. Spectral efficiency study of QC-CHPCs in multirate optical CDMA system. IEEE J. Sel. Areas Commun. 2017, 25, 118-128. [CrossRef]

16. Chen, H.C.; Chang, M.K.; Yang, G.C.; Kwong, W.C. Design of a hybrid multirate asynchronous O-CDMA system: performance, spectral efficiency, and throughput analyses. J. Opt. Commun. Netw. 2015, 7, 1034-1048. [CrossRef]

17. Chang, W.H.; Yang, G.C.; Chang, C.Y.; Kwong, W.C. Enhancing optical-CDMA confidentiality with multicode-keying encryption. J. Lightwave Technol. 2015, 33, 1708-1718. [CrossRef]

18. Abd El-Mottaleb, S.; Fayed, H.A.; Abd El-Aziz, A.; Metawee, M.A.; Aly, M.H. Enhanced spectral amplitude coding OCDMA system utilizing a single photodiode detection. Appl. Sci. 2018, 8, 1861. [CrossRef]

19. Yen, C.T.; Huang, J.F.; Zhang, W.Z. Hiding stealth optical CDMA signals in public BPSK channels for optical wireless communication. Appl. Sci. 2018, 8, 1731. [CrossRef]

20. Ahmed, M.S.; Glesk, I. Application of semiconductor optical amplifier (SOA) in managing chirp of optical code division multiple access (OCDMA) code carriers in temperature affected fibre link. Appl. Sci. 2018, 8, 715. [CrossRef]

21. Yang, C.C. The application of spectral-amplitude-coding optical CDMA in passive optical networks. Opt. Fiber Technol. 2008, 14, 134-142. [CrossRef]

22. Yang, C.C. Spectral efficiencies of the optical CDMA-based PONs using two-code keying. IEEE Commun. Lett. 2010, 14, 767-769. [CrossRef]

(C) 2019 by the author. Licensee MDPI, Basel, Switzerland. This article is an open access article distributed under the terms and conditions of the Creative Commons Attribution (CC BY) license (http://creativecommons.org/licenses/by/4.0/). 